\title{
Online Communities Administration: Defining Tools for Different Profiles
}

\author{
Elton José da Silva ${ }^{1,2}$ and Silas Sallaume ${ }^{1}$ \\ ${ }^{1}$ Departamento de Computação, Universidade Federal de Ouro Preto, \\ Campus Morro do Cruzeiro, Ouro Preto - MG, 35400-000, Brasil \\ elton@iceb.ufop.br, ssallaumedic.uff.br \\ ${ }^{2}$ Semiotic Engineering Research Group \\ Departamento de Informática, PUC-Rio, Brasil
}

\begin{abstract}
There occur a great number of breakdowns in online communities caused by the natural gap between face-to-face and virtual relationships. In addition to this shortcoming, creating, managing and promoting participative online communities is frequently arduous work, and there usually are few tools to help the administrators through this endeavor. So, in this paper we present some results of a research carried out for the implementation of a tool for managing online communities called OriOnGroups, which aims to help in the administrator's decision-making when creating and managing communities. We introduce a categorization for communities profiles and make use of personas, which helped us to select different tools for each one of these profiles.
\end{abstract}

Keywords: Online Community Administration, User/Group Profiles, Personas.

\section{Introduction}

OriOnGroups is a virtual environment for supporting online communities, developed at PUC-Rio's Department of Informatics and UFOP's Department of Computer Science. The first release of that environment basically made available a discussion forum tool for supporting discussions brought about by online academic orientation to graduate students. Subsequent releases were based on a study of expectations towards this kind of environment as evidenced by a group of potential users who were involved in academic research [2]. Over the course of this time, some of the tools included in OriOnGroups were, for instance, bulletin board, members' profiles, forum participation assessment, polls, file repository, search engines, among others. These new functionalities, which were added in a poorly planned and unstructured manner by the users themselves - Computer Science students, in Human-Computer Interaction courses -, have caused the system to become more complex and the code to become unstable. In order to resolve these setbacks, we proposed a total redesign of OriOnGroups by using an object-oriented structure and design patterns [3]. These modifications improved the OriOnGroups structure and were beneficial to its development since we had in mind a generic tool for the creation of online communities, in which the activities of defining, configuring and managing different types of communities were to be included. These features also facilitated code maintenance (by the development team) and interface configuration (by the end-users). 


\section{Online Communities Administration}

It is true that there exist many problems in present online communities, and one that can be cited is connected with the disparity between the social-virtual and social-real relationships [1], such as, for example, the way in which the hierarchical relationships among the members of a group are built. With the traditional computer architecture and programming techniques, it is difficult to build a virtual community that works in the same manner as that of a real community [2]. We believe that a way of reducing the gap between real and virtual is through a proper intervention by one or various persons in charge of an effective management of the community. By 'management' we understand a series of activities which include, for example, administration, moderation, mediation and facilitation. The goal of such intervention is to provide an environment which is more flexible and agreeable to the end-user, in such a way that the participants feel compelled to engage actively in the forums, thus making the group and the discussions more productive.

Each community has unique characteristics, requiring therefore different tools and leadership styles. However, virtual communities are designed either specifically for a certain type of group - which makes its use by another type of group inadequate - or in a very generic way - which makes it likely for a group not to realize its full potential when employing a nonspecific kind of virtual community. Unfortunately, in most existing group environments it is rather troublesome to make a change in the environment structure or in its leadership style. With the aim of defining the different types of groups which can be configured under OriOnGroups, we are utilizing a division of communities into classes as proposed by [5], as shown in Figure 1.

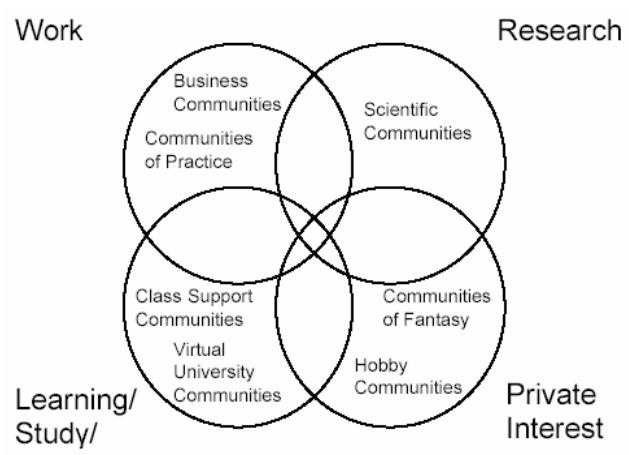

Fig. 1. Different Online Communities Profiles (extracted from [5])

The Work profile comprises communities for corporate environments and design teams. Communities of scientific research - such as those used in the early versions of OriOnGroups - could be named as typical representatives of the Research profile. Elearning communities are typical representatives of groups in the Learning/Study profile. The Private Interest profile includes entertainment communities (such as many Orkut communities) or discussion groups on private matters, such as health communities. Figure 1 also indicates that some communities can be placed in intersections of different profiles, which conveys different customization combinations at the level of the groups' administration. 


\section{Setting Up Different Types of Communities}

From the categorization of communities in [5], we began to identify and build personas - archetypical representations of real or potential users - in each presented profile. For instance, Figure 2 shows the description of a persona in a medical community domain belonging to the Research profile.

Persona: Fabio Figueiredo
Fábio is 23 years old and a medicine graduate-to-be at UFOP. During his second semester
in college he decided to specialize in Cardiology. Nowadays, he tries to keep up with the
news in his field of study, especially Chagas disease, about which he has frequent
discussions with his colleagues. He plans to take a Master's Degree at USP, where he
already participates in a research group. This is one of the reasons for his intense use of the Internet, as
he needs to maintain long-distance contact with other doctors and researchers of his group.
Fabio's goals are:
-To get ahead in academic life, acquiring knowledge in Cardiology and producing scientific articles.
-To achieve financial stability in order to marry soon.
Psychology kind: (Extroverted - Sensorial - Thinker - Judger - Planner)

Fig. 2. Excerpt of a persona's description in a typical Research community

The identification and creation of personas in the different types of community profiles has helped us to define the tools and possibilities for customization under OriOnGroups. In the Work profile, the environment shall be configured so as to make available tools like: project follow-up and management, bulletin board, individual and group appointment book, etc. Participants shall be provided with indicators of updates in group events and community usage rules. Options of moderation and inclusion of moderators shall also be allowed. The issue of information security and confidentiality shall be highly relevant in this kind of community, in which the information exchanged among participants is typically strategic to the organization. In the Research profile, the environment shall be configured so as to make available tools for helping with scientific research, such as files repository, links repository, online collaboration in article production, among others. Users shall be provided with indicators of content updates as well as quantitative and qualitative indicators related to the group as a whole and to each user, so that the production of knowledge by the group can be assessed. With regard to the community's rules, these are more flexible in this kind of environment, which has a nearly horizontal hierarchy, dispensing with the use of moderators in most cases. Here, the issue of information security and confidentiality is also important, although not as crucial as in the Work profile. Access to group knowledge is normally granted to previously invited participants only. In the Learning/Study profile, the community's administrator is provided with tools that enable him to assess each participant individually, as well as tools for publishing grades obtained by the participants in the proposed activities. In the Private Interest profile, basic tools shall be made available, such as: files and links repository, polls, photo album, blog, chat rooms, among others. Regarding the community's rules, options of moderation and inclusion of moderators shall be allowed, aiming at censoring certain themes and uploads, since, by default, any person can take part in the group.

In OriOnGroups, the group administrator can create communities under this profile classification. He/she can then configure the group, easily including and excluding 
some tools, customizing the environment to different types of groups [4]. Important is to point out that, when configuring each tool, the administrator is provided with its description and the most likely consequences of its enabling/disabling to that specific type of group (e.g.: possible effects of a chat room in a research community in which one wants to motivate more deeper discussions among participants).

\section{Conclusion}

In this paper we presented OriOnGroups, a tool for creating and configuring online communities. We believe that good tools for administering these communities can help in the job of dealing with - and possibly reducing - the gap between virtual and face-to-face group relationships. For example, it is fundamental to provide the administrators with descriptions of how the selected configurations can affect a specific type of group. Using a categorization of different online communities profiles, we created personas to help us to understand for whom we were creating a community and the types of tools to put available to its participants.

We expect these results to foster discussion on how to design more participative online community environments, and also how the use of personas can help teams in the design of interactive software. The next steps in this work will be to improve the present implementation of OriOnGroups and evaluate the management and use of virtual communities with different profiles.

Acknowledgments. "For from Him and through Him and to Him are all things. To Him be the glory forever!” (Romans 11:36)

\section{References}

1. Ackerman, M.: The Intellectual Challenge of CSCW: The gap between social requirements and technical feasibility. Human-Computer Interaction 15, 179-203 (2000)

2. da Silva, E.J, Sieckenius, C.S., Prates, R.O., Nicolaci-da-costa, A.M.: What they want and what they get: A study of light-weight technologies for online communities. In: CLIHC'2003. Proceedings of Latin-American Conference on Human-Computer Interaction, Rio de Janeiro, RJ. Conference, pp. 135-146 (2003)

3. da Silva, R.F.: Projetando uma nova estrutura para uma Comunidade Online. Trabalho de Conclusão de Curso, Departamento de Computação, UFOP, junho de (2006) (in Portuguese)

4. Sallaume, S., da Silva, R.F.: Gerenciamento em Comunidades Online: Um módulo de administração para o OriOn, XII Seminário de Iniciação Científica da UFOP (2005) (in Portuguese)

5. Seufert, S.: Design and Management of Online Learning Communities. In: Proceedings of the European Academy of Management (EURAM), Stockholm (2002) 